\title{
Optimal information encoding for multiple, simultaneously presented stimuli
}

\author{
Jan Pieczkowski ${ }^{1,2^{*}}$, Lawrence York2 ${ }^{2}$ Jeanette Hellgren Kotaleski ${ }^{1,3}$, Mark van Rossum² \\ From Twenty First Annual Computational Neuroscience Meeting: CNS*2012 \\ Decatur, GA, USA. 21-26 July 2012
}

Information in the brain is usually encoded in a way that distributes the activity over a population of neurons, referred to as population coding[1]. Population coding has been observed in almost all brain systems and renders the neural code robust, accurate, and failure resistant.

The coding of single stimuli in population codes is relatively well understood [2], and in particular the noise models, correlations, neural heterogeneity and links to psychophysics have been studied. However, the situation is much less clear when multiple stimuli are simultaneously encoded [3].

Theoretical studies (e.g., [4]) have thus far only examined linear supposition schemes that encode a probabilistic stimulus ensemble. However, experimental studies (c.f. [5], [6], [7]) suggest a non-linear encoding scheme using a maximum rule, where the response of a single neuron to a pair of stimuli equals the response to the constituent that on its own produces the maximum response, i.e.

$$
R_{A, B}=\max \left(R_{A}, R_{B}\right)
$$

We investigate the theoretical implications of these findings by comparing different encoding strategies and examine the decoding accuracy. The goal is to find the optimal encoding scheme for multiple stimuli.

We investigate the theoretical implications of these findings by comparing different encoding strategies and examine the decoding accuracy. The goal is to find the optimal encoding scheme for multiple stimuli.

In our current study, we focus on the simultaneous coding of visual stimuli representing overlapping movements of two groups of points in different directions. We investigate different ways of decoding these, among them a Maximum Likelihood decoder and estimate error rates made by these predictors, comparing to maximum rule to a linear rule.

\section{Author details}

${ }^{1}$ Department of Computational Biology, CSC, Royal Institute of Technology, Stockholm, Sweden. Department of Informatics, Edinburgh University, Edinburgh, EH8 9AB, UK. ${ }^{3}$ Department of Neuroscience, Karolinska Institutet, Stockholm, Sweden.

Published: 16 July 2012

\section{References}

1. Pouget A, Dayan P, Zemel R: Information processing with population codes. Nature Reviews Neuroscience 2000, 1(2):125-132.

2. Averbeck B, Latham P, Pouget A: Neural correlations, population coding and computation. Nature reviews Neuroscience 2006, 7(5):358-66, doi:10.1038/nrn1888.

3. Treue $\mathrm{S}, \mathrm{Hol} \mathrm{K}$, Rauber $\mathrm{H}$ : Seeing multiple directions of motion-physiology and psychophysics. Nature neuroscience 2000, 3(3):270-6, doi:10.1038/ 72985.

4. Zemel R, Dayan P: Distributional population codes and multiple motion models. Advances in Neural Information Processing Systems 1999, 11.

5. Gawne T, Martin J: Responses of primate visual cortical neurons to stimuli presented by flash, saccade, blink, and external darkening. Journal of neurophysiology 2002, 88(5):2178-86.

6. Lampl I, Ferster D, Poggio T, Riesenhuber M: Intracellular measurements of spatial integration and the MAX operation in complex cells of the cat primary visual cortex. Journal of neurophysiology 2004, 92(5):2704-13, doi:10.1152/jn.00060.2004.

7. Oleksiak A, Klink P, Postma A, van der Ham I, Lankheet M, van Wezel R: Spatial summation in macaque parietal area 7a follows a winner-take-all rule. Journal of neurophysiology 2011, 105(3):1150-8, doi:10.1152/ jn.00907.2010.

doi:10.1186/1471-2202-13-S1-P17

Cite this article as: Pieczkowski et al.: Optimal information encoding for multiple, simultaneously presented stimuli. BMC Neuroscience 201213 (Suppl 1):P17.

\footnotetext{
* Correspondence: janpi@kth.se

'Department of Computational Biology, CSC, Royal Institute of Technology, Stockholm, Sweden

Full list of author information is available at the end of the article
} 